\title{
Stress relaxation of bi-disperse polystyrene melts
}

\section{Exploring the interactions between long and short chains in non-linear rheology}

\author{
Ludovica Hengeller ${ }^{1}$, Qian Huang ${ }^{1}$, Andriy Dorokhin ${ }^{2}$, Nicolas J. Alvarez ${ }^{3}$, Kristoffer Almdal ${ }^{2}$, Ole Hassager ${ }^{1}$ \\ 1 Department of Chemical and Biochemical Engineering, Technical University of Denmark, DK-2800 Kgs. Lyngby, Denmark \\ 2 Department of Micro and Nanotechnology, Technical University of Denmark, DK-2800 Kgs. Lyngby, Denmark \\ 3 Department of Chemical and Biological Engineering, Drexel University, 3141 Chestnut Street, Philadelphia, United States
}

Received: date / Revised version: date

\begin{abstract}
We present start-up of uniaxial extension followed by stress relaxation experiments of a bi-disperse $50 \%$ by weight blend of $95 \mathrm{k}$ and $545 \mathrm{k}$ molecular weight polystyrene. We also show, for comparison, stress relaxation measurements of the polystyrene melts with molecular weight $95 \mathrm{k}$ and $545 \mathrm{k}$, which are the components of the bi-disperse melt. The measurements show three separated relaxation regimes: a fast regime, a transition regime and a slow regime. In the fast regime the orientation of the long chains is frozen and the stress relaxation is due to stretch relaxation of the short chains primarily. Conversely in the slow regime the long chains have retracted and undergo relaxation of orientation in fully relaxed short chains.
\end{abstract}

Key words polymer blend, stress relaxation, extensional flow

\section{Introduction}

Industrial polymers are largely polydisperse systems. One step towards understanding polydisperse polymers is the experimental characterization and modelling of bi-disperse blends. In fact the prediction of bi-disperse systems is a strong way to probe the validity of theoretical models.

The motion of entangled polymer chains has been described by the reptation theory of Doi and Edwards (1986), which in its original form assumes that each polymer chain slithers through a tube made of other polymer chains with a friction coefficient that is independent of the others chains. In this frame the mutual influence of reptating chains was not considered and each chain moves out of its own tube assuming an equilibrium configuration irrespective of the state of the others chains. The tube through which the polymer diffuses is assumed to be fixed in time.

Over the years many attempts were done to build blend-ing laws based on the Doi-Edwards model (Lee et al. (2005),
Khaliullin and Schieber (2010), Read et al. (2012), van Ruymbeke et al. (2012) and van Ruymbeke et al. (2014)). Two main boosts in the tube motion were proposed: a constraint released (CR) mechanism de Gennes (1975) and a dynamic tube dilation (DTD) mechanism by Marrucci (1985). The first mechanism is activated by the slow tube dynamic in which some of the entaglements' constraints along the chain are re-leased allowing more freedom in the lateral movements of the test chain. The DTD mechanism says that the tube re-newal due to the faster motion and relaxation of the surround-ing short chains results in a widening of the tube diameter speeding up the reptation of the longer chains. The fraction of the relaxed molecules acts like a solvent on the unrelaxed molecules.

The first development in the description of the CR of the double reptation mixing rule was introduced by Tsenoglou C. (1987) and des Cloizeaux (1988), which attempt to describe the terminal relaxation region, more sensitive to the bi-modal molecular weight distribution. In this approach the entanglement between two different $M_{w}$ chains is a random event proportional to the volume fraction of the components and as soon as one of the two polymers diffuses away, the cou-pling point formed by the entanglement will instantaneously relax. That holds just if the longest relaxation times of the two components are sufficiently apart from each other. Validation was found with experimental viscoelastic data combined with dielectric spectroscopy on entangled binary blends of linear cispolyisoprenes (Watanabe et al. (2004a), Watanabe et al.(2004b)).

Struglinski and Graessley (Struglinski and Graessley (1985), Struglinski and Graessley (1986)) investigated the linear viscoelasticity of five series of well entangled binary melts of linear polybutadiene, each with a wide range of concentra-tions. The ratio between the molecular weight of the long and the short molecules $M_{L} / M_{S}$ ranged between 2.5 and 10.7. Be-sides the experimental work they suggested some molecular theories, earlier based just on the tube and reptation model and later adding mechanisms such as path length fluctuations and constraint release. They introduced the existence of two 
different relaxation behaviours determined by a relevant parameter defined as $G r=M_{L} M_{e}^{2} / M_{S}^{3}$, where $M_{L}$ and $M_{S}$ are respectively the molecular weight of the long and the short component, and $M_{e}$ is the molecular weight of a strand between two entanglements. The critical value of the parameter defines the limit of the two cases. Picturing the long polymer component constraints in a tube made up of entanglements with short and long chains if $G r<<G r_{c}$, the entanglements with short chains are long-lived hence the high $M_{w}$ chain relaxes in a region constrained by both components, called thin tube, and the reptation time of the long chains is independent of blend composition. Conversely if $G r>G_{c}$ short chains constraints are not anymore active to define the long chain path and tube dilation takes place i. e. the long molecules diffuse in the wider tube where the only active constraints are within long polymers.

The exact value of $G r_{c}$ is not universally recognised. The original one proposed by Struglinski and Graessley was 1, whereas in a later work of Park and Larson (2004), was empirically fixed at 0.064 , which gave a better estimate to delimit the two regimes of the skinny and dilated tube. The authors proposed an extended version of the Milner-McLeish model (Milner and McLeish (1998)) from linear monodisperse polymers to linear bi-disperse melts incorporating dynamic tube dilation (DTD) and Rouse constraint release mechanism (CRR). The time scale is divided into three regimes: the first one until the reptation time of the short chains, an intermediate one that lasts up to the reptation of the long chains and the terminal one after the terminal relaxation of the long chains. Predictions showed good agreement with a wide series of linear viscoelastic data of bidisperse 1,4 polybutadienes and polyisoprenes linear melts spanned on a large range of $G r$ values.

Some relaxation experiments from states close to equilibrium have shown that a cooperative effect exists between chains. In particular, Kornfield et al. (1989) used a rheo-optical technique to measure the dynamic infrared dichroism in the relaxation phase following step shear for each component in bi-disperse entangled polymer melts of hydrogenated and deuterated polyisoprenes containing from 10 to $75 \%$ vol. of long chains. They found that polydispersity can strongly affect the relaxation process of the components, decreasing the longest relaxation time of the high molecular weight polymer and delaying the longest relaxation time of the short component regardless the $M_{w}$ of the long chain matrix. They explained that the observed slower relaxation of the shorter molecules is due to a combined effect of anisotropy in the orientation of the Rouse segments and a nematic orientation induced by the neighbouring long chains.

More recently, Ianniruberto et al. (2012) suggested a reduction of the monomeric friction coefficient caused by alignment of the Kuhn segments of polymer chains. The stretchorientation induced reduction of the friction $\zeta$ is based on the molecular concept that aligned objects generate an anisotropic and reduced friction when parallel to each other in contrast to the random configuration. Based on the same concept, Yaoita et al. (2012) further investigate the molecular origin of the different behavior between polymer melts and solutions with primitive chain simulations. In a more recent study on sol-vent effects in monodisperse concentrated polymer solutions, Huang et al. (2013a) also addressed to the existence of ne-matic interactions between solvent-polymer and polymer-polymer molecules to explain an observed dependence on solvent na-ture in non-linear extensional flow.

Compared to monodisperse polymer melts and solutions, the situation is more challenging for bi-disperse entangled melts because they emphasize complex interactions between long and short chains especially in non-linear relaxation pro-cess. Nonlinear viscoelastic properties in elongational flow have not been investigated as intensely. Extensional data of polystyrene blends of super high $M_{w}$ dissolved, with small volumetric fraction, in a matrix of shorter polydisperse chains were modeled by Wagner et al. (2005). The prediction showed that increasing the amount of the long component in the blends resulted in more strain hardening.

Nielsen et al. (2006) characterized three bi-disperse polystyrene melts in non-linear extensional flow, which are the only measurements that reached steady state for bi-disperse systems to our knowledge. They mixed PS-390k with PS-50k in two different concentrations to investigate the effect of dilution, as well as PS-390k with PS-100k in the same concentration of the previous one to test the effect of short molecules length. A maximum, relative to the $3 \eta_{0}$, in the steady state elongational viscosity vs. elongational rate was observed for all the samples. Conversely on the observations of Wagner et al. (2005), the amount of strain hardening increases as the long chains are more diluted. Relying on the few available extensional data several mixing laws have been developed for predicting the non-linear viscoelastic behaviour of bi-disperse linear melts (vanRuymbeke et al. (2010), Wagner (2011), Rasmussen et al. (2014).)

A more recent work by Auhl et al. (2009) focuses on the onset of chain stretch of long chains when diluted by shorter ones. Start up elongational data of two series of binary blends of polyisoprene with broad concentration range are fitted with a model that confirms their prediction: long chain stretch ap-pears at lower hencky strain when the long chains are diluted by the short ones than in the pure melt. They explain this behavior by a proposed novel mechanism, the effective stretch relaxation time, which implies the stretch relaxation of the fat tube through the motion of the chain in the thin tube.

However, data of stress relaxation following steady extensional flow, which are more relevant for the understanding of relaxation mechanisms of bi-disperse systems, are still miss-ing.

In the present work we measured a bi-disperse $50 \%$ by weight blend of $95 \mathrm{k}$ and $545 \mathrm{k}$ molecular weight poly-styrene (for which the estimated Struglinsky-Graessley parameter is $G r=0.112$ ), referred to Blend 50L-50S, in both uniaxial ex-tensional flow and stress relaxation. The purpose is to inves-tigate cooperative interactions between polymer chains and measure how the mechanism of the short chains is affected in presence of the long chains. Data for the blend are compared to data for the pure components. 
Table 1 Properties of the two monodisperse polystyrene components (the values for PS-545k are taken from Huang et al. (2013b)).

\begin{tabular}{lccccc}
\hline Sample & $M_{w}[\mathrm{~g} / \mathrm{mol}]$ & $P D I$ & $\tau_{m}[s]$ & $\tau_{R}[s]$ & $Z$ \\
\hline PS-545k & 545000 & 1.12 & 58750 & 705 & 41.0 \\
PS-95k & 95100 & 1.07 & 169.3 & 20.4 & 7.14 \\
\hline
\end{tabular}

\section{Experimental}

\subsection{Synthesis and chromatography}

The polystyrene PS-545k melt used in this work has been previously described and characterized in both shear and extensional rheology by Huang et al. (2013b). The polystyrene PS-95k has been synthesized by the means of living anionic polymerization according to the standard procedure by Ndoni et al. (1995). The reaction was carried out for 4 hours at 35 ${ }^{\circ} \mathrm{C}$ in freshly distilled cyclohexane, with the use of the titrated solution of sec-butyllithium in hexane as the initiator. The molar mass of PS-95k was determined with size exclusion chromatography (SEC) with non-stabilized tetrahydrofuran (THF) as the eluent and with the use of a column set consisting of a $5 \mu \mathrm{m}$ guard column and two $300 \times 8 \mathrm{~mm}^{2}$ columns (PLgel Mixed C and Mixed D). The system was equipped with a triple detector system including a combined Viscotek model 200 differential refractive index (DRI), a differential viscosity detector and a Viscotek model LD 600 right angle laser light scattering detector (RALLS). On the basis of calibration with narrow molar mass polystyrene standards and flow rate signal adjusting according to Irganox signals, the values of the weight-average molecular weight $\bar{M}_{w}$ and the polydispersity index $P D I$, defined as the ratio of the $\bar{M}_{w}$ over the number-average molecular weight $\bar{M}_{n}$, were determined. Table 1 summarizes the weight-average molecular weight $\bar{M}_{w}$ and the polydispersity index $P D I$ of the two polystyrenes.

\subsection{Blend preparation}

The polystyrene blend was prepared using the two monodisperse melts described above. PS-95k and PS-545k were dissolved together in THF and the solution was stirred at room temperature for 24 hours. Once both components were well dissolved and mixed homogeneously, the THF solution was poured into methanol drop by drop, which precipitated out the blend. The precipitated blend was recovered by filtration. The wet powder was then dried for more than eight hours in open air and later dried in a vacuum oven at $70^{\circ} \mathrm{C}$ for 2 weeks to ensure the complete evaporation of the residual solvent. To check concentration homogeneity two samples at different locations of the blend were tested in SEC. The concentration analysis was conducted by integral estimation of the peak areas of the bimodal curve in SEC to make sure that the desired concentration was obtained.

Table 2 summarizes the corresponding weight fraction of the components in the bi-disperse blend 50L-50S. The weight-
Table 2 The weight fractions of PS-95k and PS-545k and the weight average molecular weight $\bar{M}_{w}[\mathrm{~g} / \mathrm{mol}]$ of the Blend 50L-50S.

\begin{tabular}{lccc}
\hline Sample & $w t \% P S-545 k$ & $w t \% P S-95 k$ & $\bar{M}_{w}[\mathrm{~g} / \mathrm{mol}]$ \\
\hline Blend 50L-50S & 49.8 & 50.2 & 319000 \\
\hline
\end{tabular}

average molecular weight $\bar{M}_{w}$ has also been calculated for the blend and the value is reported in the same table.

\subsection{Mechanical spectroscopy}

The linear viscoelasticity of the pure monodisperse poly-styrene melts and the Blend 50L-50S have been measured on an ARESG2 rheometer from TA Instruments, with an $8 \mathrm{~mm}$ parallel plate geometry. Small amplitude oscillatory shear flow measurements were performed for all three samples at 130, 150 and $170^{\circ} \mathrm{C}$ in nitrogen atmosphere. The data were shifted to a single master curve at $130^{\circ} \mathrm{C}$, using the principle of time temperature superposition (TTS). The time-temperature shift factors for PS-95k, PS-545k and the Blend 50L-50S were found to be in agreement with a single Williams-LandelFerry (WLF) equation of the form,

$$
\log _{10} a_{T}=\frac{-c_{1}^{0}\left(T-T_{0}\right)}{c_{2}^{0}+\left(T-T_{0}\right)}
$$

where $c_{1}^{0}=8.99$ and $c_{2}^{0}=81.53, T_{0}=130^{\circ} \mathrm{C}$ and $\mathrm{T}$ is the temperature in ${ }^{\circ} \mathrm{C}$.

\subsection{Extensional flow}

The extensional stress measurements were performed with a filament stretching rheometer (FSR) (Bach et al. (2003b), Román Marín et al. (2013)). The FSR has been recently modified with the introduction of two independent motors, one connected to the top plate and the other one connected to the laser. Before starting an experiment, the samples of Blend 50L-50S were molded into cylindrical shaped pillars with a fixed radius $R_{0}$ of $2.7 \mathrm{~mm}$ and a thickness of $L_{0}$ between 1.32 and $1.45 \mathrm{~mm}$, giving an aspect ratio $\Lambda_{0}=L_{0} / R_{0}$ ranging between 0.49 and 0.54 . The same procedure was followed for the monodisperse PS-95k samples, except that the radius $R_{0}$ was $4 \mathrm{~mm}$ and the thickness $L_{0}$ was around $2 \mathrm{~mm}$ for all the samples. To make sure that no air bubbles were trapped in the samples, the dried polymer powder was molded at approximately $150^{\circ} \mathrm{C}$ for 20 minutes and then pressed and annealed at the same temperature under vacuum for an additional 20 minutes, to ensure the complete relaxation of the polymer chains. Before each experiment the sample was allowed to fully relax after pre-stretching to a radius $R_{p}$. The Hencky strain is calculated from on line measurement of the midfilament radius $R(t)$ as

$$
\varepsilon=-2 \ln \left[R(t) / R_{p}\right]
$$


while the strain rate is defined as $\epsilon^{\circ}=d \epsilon / d t$. The mean value of the difference between the axial and the radial stress in the mid-filament is obtained from the measured values of $F$ $(t)$ and $R(t)$, with a force balance that neglects surface tension and inertial effects as reported by Szabo (1997) and Szabo and McKinley (2003) as

$$
\left\langle\sigma_{z z}-\sigma_{r r}\right\rangle=\frac{F(t)-m_{f} g / 2}{\pi R(t)^{2}},
$$

where $F(t)$ is the axial force, $g$ is the gravitational acceleration and $m_{f}$ is the weight of the filament. At small strains, during the start up of the extensional flow, there is a contribution in the stress difference that arises from the shear components in the deformation field. This effect is caused by the noslip boundary condition at the end plates, and is more prominent at small aspect ratios (Nielsen et al. (2006)). This effect may be compensated by a correction factor as mentioned in Rasmussen et al. (2010) that ensures less than 3\% deviation from the corrected initial stress. For large strains the correction vanishes and the radial variation of the stress in the symmetry plane becomes negligible (Kolte et al. (1997)). Finally, the extensional stress growth coefficient is calculated as

$$
\bar{\eta}^{+}=\frac{\left\langle\sigma_{z z}-\sigma_{r r}\right\rangle}{\epsilon} .
$$

Stress relaxation measurements present a special challenge, since merely halting the plates does not produce a true stress relaxation experiment, because typically the filament will be subjected to a progressive thinning on its own (Wang et al. (2007); Lyhne et al. (2009)). A technique to perform a di-rect stress relaxation experiment following steady uniaxial extension on polymer melts has been introduced by Nielsen et al. (2008) on the above-mentioned FSR. The novelty consists in the use of a closed loop controller that monitors the mid-filament diameter; in this way the necking is avoided by adjusting the position of the top plate. In a stress relaxation experiment, at the start-up of the elongation, a constant strain rate is applied on the sample. As soon as the stress relax-ation starts at an arbitrarily given Hencky strain $\epsilon_{0}$, the midfilament radius is kept constant by the active control loop, giving $\epsilon^{*}=0$. The extensional stress decay coefficient during the stress relaxation is defined as

$$
\bar{\eta}^{-}=\frac{\left\langle\sigma_{z z}-\sigma_{r r}\right\rangle}{\epsilon},
$$

where $\dot{\boldsymbol{e}} \mathrm{s}$ the strain rate in the start-up of the uniaxial extensional flow. The extra shear contribution, mentioned before for the start-up of the extension flow, is negligible during the stress relaxation process (Nielsen et al. (2008)).

\section{Results and analysis}

\subsection{Linear viscoelasticity}

The storage modulus $G^{\prime}$ and loss modulus $G^{\prime \prime}$, plotted as a function of the angular frequency $\omega$, are shown in Figure 1 for
PS-95k and PS-545k, and in Figure 2 for the Blend 50L-50S. The LVE data for the two monodisperse melts have been fit-ted with a continuous Baumgaertel-Schausberger-Winter (BSW) relaxation spectrum (Baumgaertel et al. (1990)). The choice of using this model is due to its success in describing lin-ear monodispese solutions and melts (Huang et al. (2013a), Huang et al. (2013b)). The stress relaxation modulus $G(t)$ is defined in terms of the continuous spectrum $H(\tau)$ of relax-ation times $\tau$ as follows,

$$
\begin{gathered}
G(t)=\int_{0}^{\infty} \frac{H(\tau)}{\tau} \exp (-t / \tau) d \tau, \\
H(\tau)=H_{e}(\tau)+H_{g}(\tau) .
\end{gathered}
$$

The spectrum $H(\tau)$ is composed of two contributions due to two different behaviours: the entanglement or viscoelastic one, defined as $H_{e}(\tau)$; and the glassy one, defined as $H_{g}(\tau)$, expressed as

$$
\begin{aligned}
& \left.H_{e}(\tau)=n_{e} G_{N}^{0} \frac{\tau}{\tau_{m}}\right)^{n_{e}} h\left(1-\tau / \tau_{m}\right), \\
& \left.H_{g}(\tau)=n_{e} G_{N}^{0} \frac{\tau}{\tau_{c}}\right)^{-n_{g}} h\left(1-\tau / \tau_{m}\right) .
\end{aligned}
$$

Here $G_{N}^{0}$ is the plateau modulus, $\tau_{c}$ is the characteristic time of the glassy mode, $\tau_{m}$ is the longest relaxation time, $n_{e}$ and $n_{g}$ are the slopes, respectively, of the viscoelastic and the glassy spectra in a log-log plot. $h(x)$ is the Heaviside step function determining the cut-off of the two spectra. The val-ues of $n_{e}$ and $n_{g}$, which are material specific constants, were fixed to 0.23 and 0.70 , respectively, in the case of nearly monodisperse polystyrene melts (Huang et al. (2013a), Huang et al. (2013b)), while the other three parameters $G_{N}^{0}$, $\tau_{c}$ and $\tau_{m}$ were optimized by the fitting. A detailed explanation of the meaning of BSW-parameters can be found in Huang et al.(2013b). For convenience we reproduce here the asymptotic result in the glassy regime

$$
\begin{gathered}
G^{\prime \prime}(\omega) /\left(G_{N}^{0} n_{e}\right) \sim \frac{\pi}{2 \cos \left(n_{g} \pi / 2\right)}\left(\tau_{c} \omega\right)^{n_{g}} \text { for } \omega \rightarrow \infty, \\
\tan \delta=G^{\prime \prime} / G^{\prime}=\tan \left(n_{g} \pi / 2\right) .
\end{gathered}
$$

Correspondingly it may be shown that the relaxation modulus in the glassy regime $\left(t \ll \tau_{m}\right)$ has the power law behaviour

$$
G(t) /\left(G_{N}^{0} n_{e}\right)=\Gamma\left(n_{g}\right)\left(t / \tau_{c}\right)^{-n_{g}},
$$

where $\Gamma()$ is the Gamma function.

The material properties of the two PS mono-disperse melts obtained from the BSW-fitting of the master curve at $130^{\circ} \mathrm{C}$ are listed in Table 3, together with the value of the zeroshear-rate viscosity $\eta_{0}$ and an average relaxation time $\tau_{w}$ (Nielsen et al. (2006)) calculated as

$$
\eta_{0}=\int_{0}^{\infty} G(s) d s \doteq n_{e} G_{N}^{0} \tau_{m},
$$


Table 3 Material properties of PS-95k and PS-545k obtained from the BSW-fitting. Values for PS-545k are taken from Huang et al. (2013b).

\begin{tabular}{lcc}
\hline Parameters & PS-545k & PS-95k \\
\hline$n_{e}$ & 0.23 & 0.23 \\
$n_{g}$ & 0.7 & 0.7 \\
$G_{N}^{0}[\mathrm{~Pa}]$ & 256700 & 258080 \\
$\tau_{c}[\mathrm{~s}]$ & 0.419 & 0.400 \\
$\tau_{m}[\mathrm{~s}]$ & 58750 & 169.3 \\
$\tau_{w}[\mathrm{~s}]$ & 32400 & 93.38 \\
$\eta_{0}[\mathrm{~Pa} \cdot \mathrm{s}]$ & $2.823 \cdot 10^{9}$ & $8.827 \cdot 10^{6}$ \\
\hline
\end{tabular}

Table 4 Material parameters of PS-95k and PS-545k obtained from the ML-theory.

\begin{tabular}{lcc}
\hline ML Parameters & PS-545k & PS-95k \\
\hline $\bar{M}_{e}[\mathrm{Kg} / \mathrm{mol}]$ & 17.1 & 16.8 \\
$G_{e}[\mathrm{~Pa}]$ & $3.41 \cdot 10^{5}$ & $3.44 \cdot 10^{5}$ \\
$\tau_{e}[\mathrm{~s}]$ & 0.41 & 0.67 \\
$c_{v}[\mathrm{~s}]$ & 0.1 & 0.1 \\
\hline
\end{tabular}

$$
\tau_{w}=\frac{\int_{0}^{\infty} G(s) s d s}{\int_{0}^{\infty} G(s) d s} \doteq \frac{1+n_{e}}{2+n_{e}} \tau_{m} .
$$

In both equations the dot indicates that the glassy contributions have been neglected. Note the value of the parameter $n_{g}$ $=0.70$ (corresponding to $\tan \delta \approx 2$ ) as the best fit to the data. It reflects the slope of $G^{\prime \prime}(\omega)$ for large frequency. This regime, commonly referred to as the Rouse regime has been extensively analysed (Likhtman et al. (2002)) in terms of constrained and unconstrained Rouse motion. We include therefore in Figure 1 the McLeish-Likhtman (ML) theory predictions Table 4. The prediction is almost identical with the BSW fitting except in the limit of large frequencies, where the ML-theory shows $G^{\prime}(\omega) \sim G^{\prime \prime}(\omega)$ in contrast to the data. For the blend we use the Multi-mode Maxwell spectrum in the form

$$
G(t)=\sum_{k} g_{k} e^{-t / \lambda_{k}}
$$

The Maxwell fitting parameters $g_{k}$ and $\lambda_{k}$ for the Blend 50L$50 \mathrm{~S}$ are reported in Table 5. The value of the zero-shear-rate viscosity and average relaxation time computed from the definitions in Eqs. 13 and 14 become respectively $\eta_{0}=\sum \lambda_{k} g_{k}=$ $3.82 \cdot 10^{8} \mathrm{~Pa} \mathrm{~s}$ and $\tau_{w}=\sum \lambda_{k}^{2} g_{k} / \sum \lambda_{k} g_{k}=2.3 \cdot 10^{4} \mathrm{~s}$.

Comparing the average relaxation time $\tau_{w}=23000 \mathrm{~s}$ for the Blend 50L-50S with the one obtained from the BSW fitting of the LVE data of the pure 545k (long component) $\tau_{w}=$ 32400 s, both computed with Eq. 14, we can affirm that the terminal relaxation of the long chains in the blend is somewhat faster than in the pure melt. We take this to mean that the long chains are relaxing in a fat tube where the short chains are not imposing significant constraints. In the frame of the Struglinsky-Graessley criterion we are consequently in
Table 5 Maxwell fitting parameters $g_{k}$ and $\lambda_{k}$ for the Blend 50L$50 \mathrm{~S}$.

\begin{tabular}{lcc}
\hline $\mathrm{k}$ & $g_{k}(\mathrm{~Pa})$ & $\lambda_{k}(\mathrm{~s})$ \\
\hline 1 & $6.76 \cdot 10^{3}$ & $3.43 \cdot 10^{4}$ \\
2 & $1.83 \cdot 10^{4}$ & $6.31 \cdot 10^{3}$ \\
3 & $1.52 \cdot 10^{4}$ & $1.16 \cdot 10^{3}$ \\
4 & $6.67 \cdot 10^{4}$ & $2.14 \cdot 10^{2}$ \\
5 & $5.70 \cdot 10^{4}$ & $3.93 \cdot 10^{1}$ \\
6 & $5.28 \cdot 10^{4}$ & 7.22 \\
7 & $7.08 \cdot 10^{4}$ & 1.33 \\
8 & $1.84 \cdot 10^{5}$ & $2.44 \cdot 10^{-1}$ \\
9 & $4.06 \cdot 10^{5}$ & $4.50 \cdot 10^{-2}$ \\
10 & $1.15 \cdot 10^{6}$ & $8.27 \cdot 10^{-3}$ \\
11 & $3.23 \cdot 10^{6}$ & $1.52 \cdot 10^{-3}$ \\
12 & $5.14 \cdot 10^{7}$ & $2.80 \cdot 10^{-4}$ \\
\hline
\end{tabular}

the limit of $G r>G r_{c}$. Our system $(G r=0.112)$ therefore seems to agree with the value of 0.064 reported by Park and Larson (2004) rather than the value $G r_{c}=1$ reported by Struglinski and Graessley (1985), Struglinski and Graessley (1986).

\subsection{Start up of extensional flow}

The molecular weight $M_{w}$ of the two monodisperse components in Blend 50L-50S is selected such that their respective longest relaxation times are well separated from each other. We expect therefore that different mechanisms can be activated for each component independently. The Rouse time $\tau_{R}$, which indicates the stretch relaxation time, is defined as $\tau_{R}=Z^{2} \tau_{e}$, where $\tau_{e}$ is the relaxation time of the strand between two entanglements with molecular weight $M_{e}$, and $Z=$ $M_{w} / M_{e}$ is the number of entanglements per chain. We use $M_{e}=13300 \mathrm{~g} / \mathrm{mol}$ (Bach et al. (2003a)) to determine the value of $Z$ for our monodisperse melts. The reptation time $\tau_{m}$, which indicates the maximum relaxation time of a whole chain, can be obtained from LVE measurements. The values of $\tau_{m}, \tau_{R}$ and $Z$ for the two monodisperse polystyrenes are listed in Table 1.

To identify the regimes, we wish to interpret the dynamics in terms of time constants for the components. We estimate the Rouse times of the short and long constituents in the blend to be identical to those in the pure melts given in Table 1. Moreover since the long chains are essentially frozen at the relaxation time of the short component, we may roughly estimate the terminal relaxation time of the short chain to be approximately equal to that of the pure melt. However, the terminal relaxation of the blend will be reptation of the long chains in a sea of fully relaxed short chains. As an estimate for this time we use the relaxation time $\tau_{w}$ obtained from the Maxwell spectrum. Hence we arrive at the relaxation times in Table 6.

To relate the relaxation in terms of the molecular parameters of the two components, we discuss the strain rates in terms of their time constants. Based on the relaxation times from Table 6 we define non-dimensional stretch rates in terms of the 
Table 6 Time constants for short and long components in the blend.

\begin{tabular}{lcc}
\hline Blend 50L-50S & Rouse time & Terminal time \\
\hline Short component & $\tau_{R}^{S}=20.4 \mathrm{~s}$ & $\tau_{m}^{S}=169 \mathrm{~s}$ \\
Long component & $\tau_{R}^{L}=705 \mathrm{~s}$ & $\tau_{w}^{L}=23000 \mathrm{~s}$ \\
\hline
\end{tabular}

Table 7 Weissenberg numbers based on Rouse time and terminal time for short and long chains.

\begin{tabular}{lcccc}
\hline$\dot{\epsilon}\left[s^{-1}\right]$ & $W i_{R}^{S}$ & $W i_{d}^{S}$ & $W i_{R}^{L}$ & $W i_{d}^{L}$ \\
\hline $1 \cdot 10^{-5}$ & $2.04 \cdot 10^{-4}$ & $1.69 \cdot 10^{-3}$ & $7.05 \cdot 10^{-3}$ & 0.23 \\
$3 \cdot 10^{-4}$ & $6.12 \cdot 10^{-3}$ & 0.012 & 0.21 & 6.9 \\
0.003 & 0.061 & 0.51 & 2.1 & 69 \\
0.03 & 0.61 & 5.1 & 21 & 690 \\
0.1 & 2.0 & 17 & 71 & 2300 \\
\hline
\end{tabular}

Weissenberg number $W i=\dot{\epsilon} \tau$ for the short and long chains as reported in Table 7, where the superscripts $S$ and $L$ refer to short and long chains respectively.

Figure 3 shows the measured corrected extensional stress growth coefficient $\bar{\eta}^{+}$plotted as a function of time at $130{ }^{\circ} \mathrm{C}$ for the Blend 50L-50S. The sample was stretched at 5 different rates $\dot{\epsilon}$, which correspond to 5 different flow regions separated by the reptation time and Rouse time of the long and short chains. All the measurements were conducted at $130^{\circ} \mathrm{C}$, except the ones at the slowest stretch rates $\dot{\epsilon}=1 \cdot 10^{-5} \mathrm{~s}^{-1}$ and $\dot{\epsilon}=3 \cdot 10^{-4} s^{-1}$. These two were performed at $160{ }^{\circ} \mathrm{C}$ and shifted to $130{ }^{\circ} \mathrm{C}$ according to the Time Temperature Superposition principle, with shift factors $a_{T}=0.0038$ and $b_{T}=$ 0.91. The solid line in the plot is the LVE prediction calculated from the parameters listed in Table 5. The elongational measurements show good agreement with the LVE at small strains. The measurement at $\dot{\epsilon}=1 \cdot 10^{-5} s^{-1}$ closely follows the LVE, except at high Hencky strains where the stress is slightly higher. At this strain rate we expect the system to be still close to the equilibrium configuration, since $\dot{\varepsilon}<1 / \tau_{d}^{L}$ which means that the flow is not fast enough to orient the polymer chains. As the $\dot{\epsilon}$ increases, more and more devia-tion from the LVE envelope of the experimental curves is observed, meaning that the chains experience higher degree of stretching, which results in more strain hardening. Even at $\dot{\epsilon}$ $=3 \cdot 10^{-4} s^{-1}$, corresponding to a $W i_{R}^{L}$ of 0.2 , light strain

hardening is observed. This is in fact in agreement with the work of Auhl et al. (2009) who predict an increase in the stretch relaxation time of the pure long chains when diluted by shorter chains. From Table 7 it can be seen that only for the highest stretch rate $\left(\dot{\epsilon}=0.1 s^{-1}\right) W i_{R}^{S}>1$, indicating the short chains are stretched by the flow. In Figure 4 we show the same corrected extensional stress difference $\left\langle\sigma_{z z}-\sigma_{r r}\right\rangle$ plotted as a function of Hencky strain at $130^{\circ} \mathrm{C}$ for the Blend 50L-50S. From this plot it is clear that $\left\langle\sigma_{z z}-\sigma_{r r}\right\rangle$ reaches a steady-state value above $\epsilon=3$ for each stretch rate. That allows the determination of a steady-state viscosity $\bar{\eta}_{s}$.

\subsection{Relaxation after fixed flow time}

The uniaxial extensional flow was stopped in the start-up after a fixed flow time of $t_{0}=35 \mathrm{~s}$ and then stress relaxation was performed. Figure 5 shows the measurements at $130^{\circ} \mathrm{C}$ at $\dot{\epsilon}=0.003,0.03$ and $0.1 \mathrm{~s}^{-1}$. The Weissenberg numbers corresponding to the three strain rates $W i_{R}^{S}=0.061,0.61$ and 2 and $W i_{R}^{L}=2.1,21$ and 71, meaning that the long chains are in all experiments stretched by the flow while the short components are near to equilibrium at the slowest rate, oriented at $0.03 \mathrm{~s}^{-1}$ and stretched at the highest strain rate.

To analyze the data let us initially recall the LVE analysis for the Maxwell spectrum of the form in Eq. 15. If we shift the time axis such that the flow is from $t=-t_{0}$ where $t_{0}=\epsilon_{0} / \dot{\epsilon}$ to $t=0$, the stress decay for $t>0$ is given in the linear viscoelastic limit by

$$
\sigma_{E}^{-}(t)=\dot{\varepsilon} \sum_{j} g_{j} \lambda_{j}\left(1-e^{-t_{0} / \lambda_{j}}\right) e^{-t / \lambda_{j}}
$$

It is illustrative to consider the two limits $t_{0} \rightarrow 0$ (relaxation after step strain), with $t_{0} \cdot \dot{\varepsilon}=\varepsilon$ fixed, and $t_{0} \rightarrow \infty$ (relaxation after steady flow):

$$
\begin{aligned}
& \text { step strain for } t_{0} \rightarrow 0: \quad \sigma_{E}^{-}(t)=\varepsilon \sum_{j} g_{j} e^{-t / \lambda_{j}} \\
& \text { steady flow for } t_{0} \rightarrow \infty: \quad \sigma_{E}^{-}(t)=\dot{\varepsilon} \sum_{j} g_{j} \lambda_{j} e^{-t / \lambda_{j}}
\end{aligned}
$$

By $t_{0} \rightarrow \infty$ and $t_{0} \rightarrow 0$ we mean $t_{0}$ larger than the longest relaxation time and smaller than the shortest relaxation time respectively. Thus for $t_{0}<t_{\min }$ the relaxation modes are weighted according to the individual moduli irrespective of the time constants. Conversely for relaxation after $t_{0}>t_{\max }$ the contributions from the individual modes are weighted by the products of the moduli and the time constants. Hence the fast modes from the small time constants contribute only a negligible amount compared to the longest time constants for relaxation after steady flow. Moreover the LVE prediction of stress decay depends on the strain rate applied in the uniaxial flow according to Eq. 18. In order to show the relaxation process at earlier times we take the stress decay data from Figure 5 and plot them according to Eq. 16 in Figure 6. For $\dot{\epsilon}=0.003 \mathrm{~s}^{-1}$ and $\dot{\epsilon}=0.03 \mathrm{~s}^{-1}$, at short times (first $10 \mathrm{~s}$ approximately) the stress for Blend 50L-50S is almost constant. At the same early times, the corresponding relaxation after the experiment at $\dot{\epsilon}=0.1 \mathrm{~s}^{-1}$ is much faster.

\subsection{Stress relaxation following steady extensional flow}

We performed stress relaxation measurements after a fixed Hencky strain of 3.5, where steady-state was reached. The three strain rates are the same as the experiments at fixed flow time. 
Figure 7 and 8 show the results for the Blend 50L-50S and PS-95k, respectively. In Figure 7 we plot the extensional stress growth coefficient $\bar{\eta}^{+}(t)$ followed by the stress decay coefficient $\bar{\eta}^{-}(t)$ as a function of time for the Blend 50L-50S. All the measurements were performed at $130^{\circ} \mathrm{C}$. We expected steady extensional flow to be established at $\epsilon_{0}=3$, see Fig. 4. To confirm this hypothesis one additional experiment was performed with $\dot{\epsilon}=0.1 \mathrm{~s}^{-1}$ and $\epsilon_{0}=4$. Indeed it appears that the relaxation process is independent of $\epsilon_{0}$ provided $\epsilon_{0}>3$, confirming that once steady flow is reached, the following relaxation process is unaffected by the flow time. Also in the plot we show an experiment made at $\dot{\epsilon}=0.003 \mathrm{~s}^{-1}$ and stopped at $\epsilon_{0}=0.3$, so that the relaxation spectrum is in the linear regime. This measurement agrees completely with the LVE predictions shown by the yellow solid line in fig-ure 7. The black solid line in the figure 7 is the LVE enve-lope for start-up. Figure 8 shows the analogous results for the monodisperse melt PS-95k. At the stretch rate of $\dot{\epsilon}=0.003 \mathrm{~s}^{-1}$ $\left(W i_{d}^{S}=0.51\right)$, the measured data follow the LVE envelope and the relaxation prediction (dashed line) even at Hencky strain of 3.5. Figures 9, 10 and 11 compare the stress relaxation process of the Blend 50L-50S with the pure short chain PS-95k at the same three strain rates and the same im-posed macroscopic Hencky strain. In Figure 9 concurrently with the stress decay data of the Blend 50L-50S and the PS-95k we also show the measurement of the pure long com-ponent in the blend, PS-545k. Data points for $t>600 s$ have not been reported because their reproducibility ends up at that time (Figure 9). The very high $M_{w}$ of the sample prevented measurements at higher strain rates. Although the short components represent $50 \%$ in weight of the blend it is seen from Figure 9 that the stress in the Blend 50L-50S is much closer to the stress in the pure long component than the stress in the pure short component. It means that the short chains do not contribute significantly to the stress level (depicted by the dashed lines in the plot) in the bi-disperse melt. The stress reduction in the Blend 50L-50S relative to the pure long component is about a factor of 2 . Also in figure 9 we have shifted the pure long component stress vertically to compare with the blend. It appears that the initial relaxation for the blend is somewhat slower than for the pure component. This may be related to the increase in the relaxation time due to dilution with short chains predicted by Auhl et al. (2009).

Figures 10 and 11 show comparisons of the stress decay data of the Blend 50L-50S and the PS-95k after cessation of steady elongational flow at the strain rate respectively of $\dot{\epsilon}=0.03 \mathrm{~s}^{-1}$ and $\dot{\epsilon}=0.1 \mathrm{~s}^{-1}$. Also here the low molecular weight component in the blend does not seem to have a significant contribution in the stress of the blend. It would be interesting to compare the orientation of the short chains in the blend and in the pure short chain melt on the molecular level. The purpose of this comparison would be to determine if the short polymers are independent from the long ones or there is some kind of interaction, e.g. that the presence of the long chains in the blend might activate a higher degree of stretching of the short molecules. The right tool that can cast light on this question is neutron scattering on quenched PS samples (Hayes et al. (1996)).

Concerning the measurements of the Blend 50L-50S at different strain rates we refer to Figure 12. The stress relaxation curves can be approximately divided into three regimes: a fast dynamic regime covering time from start of relaxation to about $20 s$, a transition regime from $20 s$ to about $700 s$ and a slow dynamics regime from $700 s$ to the end of the experiments which is well before the terminal time of the long chains. Initially we concentrate on the relaxation for time up to $20 \mathrm{~s}$. This number corresponds closely to the Rouse time of the short chains. Hence we suggest that the molecular relaxation is dominated by fast stretch relaxation of both the long and the short components. The large slope in this region and the knee at 20s supports this expectation. The transition regime from about $20 s$ to $700 s$ would then be retraction of the long chains in a sea of reptating short chains. The relax-ation up to $20 s$ is faster after extension at the highest stretch rate as observed also by Nielsen et al. (2008). This seems nat-ural since more modes are presumably excited at the highest stretch rates. Keep in mind however, that for fixed $\dot{\epsilon}_{0}$, the flow time decreases with increasing extension rate. According to Eqs. 16-17 a decrease in the flow time would in itself result in faster relaxation even in the LVE limit. The long time dy-namics for $t \sim 700 \mathrm{~s}$, is dominated by a power law behaviour over two decades. In this regime we expect relaxation of the long chains in a sea of essentially equilibrated short chains. Moreover there is a slight vertical shift here, which can be attributed to the disentanglement of the long chains by the steady flow prior to relaxation. The trend is in the right way, higher strain rates give rise to more disentanglement, up to order $50 \%$ as predicted by Andreev et al. (2013). To spec-ulate on the origin of the power law behaviour, we note that the short chains are completely relaxed, and the long chains have completely retracted-only anisotropic orientation of the long chains remains. The long chains begin therefore to un-dergo their usual, equilibrium relaxation processes, as char-acterized by the LVE data in Figure 2. Indeed we note from Fig 2 a Rouse like power law behaviour up to frequencies around $2 \cdot 10^{-3}$ corresponding in time roughly to the power law stress relaxation for $t \sim 700 \mathrm{~s}$.

\section{Conclusions}

Results are given for a fixed flow time prior to relaxation and for relaxation after steady flow. In both situations we observe that the rate of stress relaxation increases as the stretch rate in the prior flow is increased.

The measurements are analyzed in terms of three separated relaxation regimes: a fast regime, a transition regime and a slow regime. In the fast regime we expect the orientation of the long chains to be essentially frozen and the stress relaxation to be due to stretch relaxation of the short chains primarily. Conversely in the slow regime we expect the long chains to have retracted and to be undergoing relaxation of orientation in a sea of essentially fully relaxed short chains 


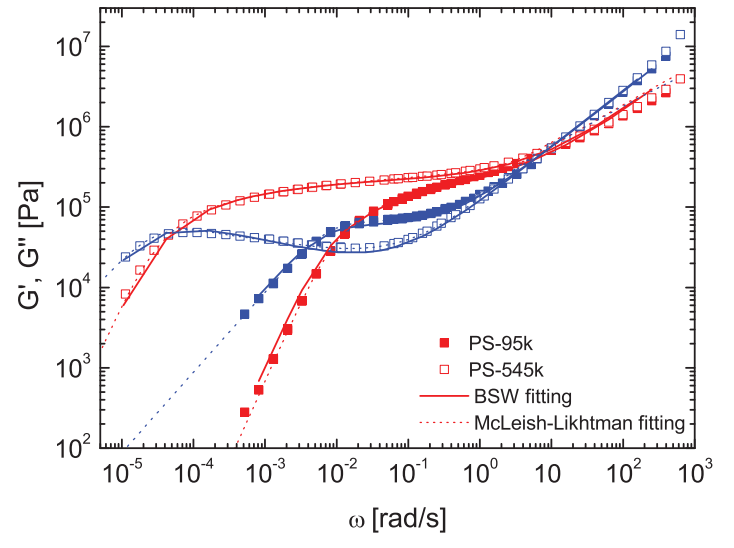

Fig. 1 LVE data fitted with the BSW spectrum and the McLeishLikhtman theory for PS-545k and PS-95k at $130{ }^{\circ} \mathrm{C}$.

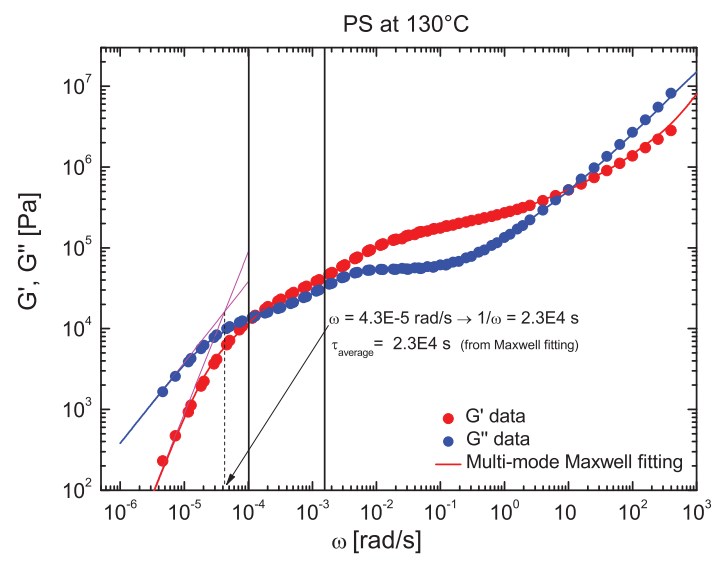

Fig. 2 LVE data fitted with the Multi-mode Maxwell spectrum and the McLeish-Likhtman theory for Blend 50L-50S at $130^{\circ} \mathrm{C}$.

that act as a solvent. The latter relaxation has a distinct power law behaviour that extends in one situation over more than two decades in time. It is noted that the power law corresponds to a similar relaxation in the low frequency part of the linear viscoelastic spectrum, although this correspondence may be fortuitous. Comparison with the pure long and short components seems to indicate that the stress in the blend is carried primarily by the long component even though it represents only $50 \%$ by weight.

Acknowledgements The research leading to these results has received funding from the Danish Council for Independent Research Natural Sciences under EPMEF on Grant 0602-02179B, and Aage og Johanne Louis-Hansens Fond. We would like to thank the anonymous reviewers for constructive observations and suggestions.

\section{References}

Auhl D, Chambon P, McLeish TCB, Read DJ (2009) Elongational Flow of Blends of Long and Short Polymers: Effec-

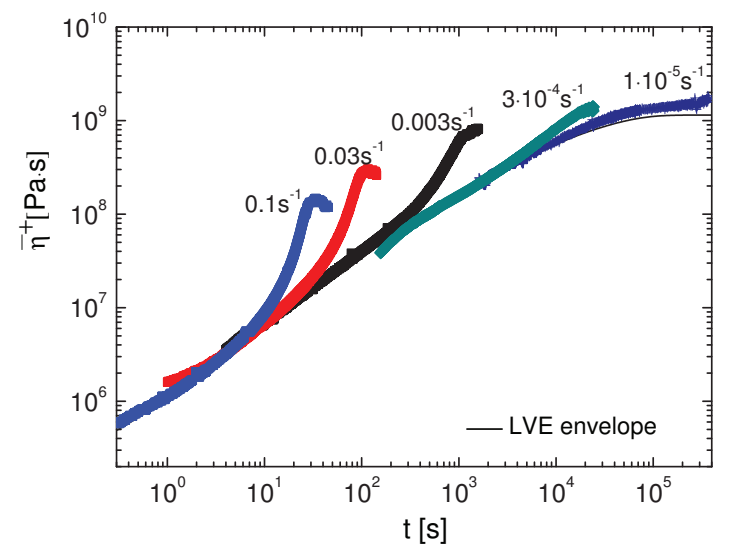

Fig. 3 Stress growth coefficient as function of time for the Blend 50L-50S measured at $130^{\circ} \mathrm{C}$. Except for the two longest stretch rates measured at $160{ }^{\circ} \mathrm{C}$ and then shifted to $130^{\circ} \mathrm{C}$ with the horizontal shift factor $a_{T}=0.0038$ and the vertical shift factor $b_{T}=0.91$, according to the TTS superposition principle. The solid line is the LVE prediction based on the parameters listed in Table 5.

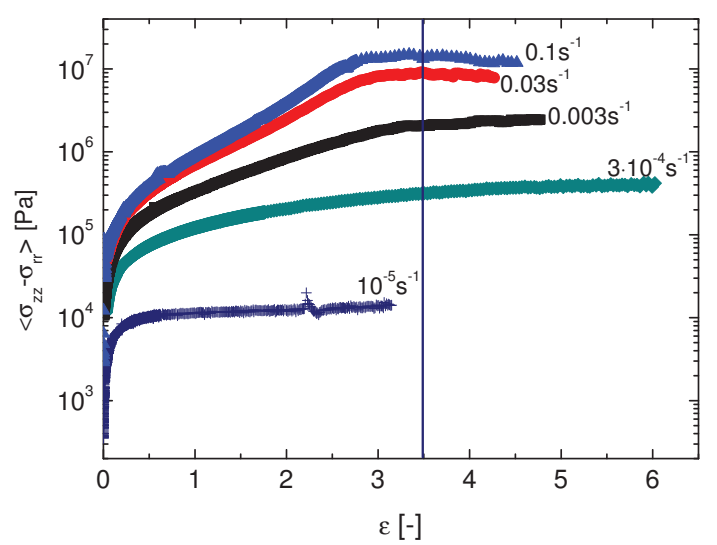

Fig. 4 The measured stress for the Blend 50L-50S at $130^{\circ} \mathrm{C}$ as function of Hecky strain. The two slowest stretch rate measurements were performed at $160{ }^{\circ} \mathrm{C}$ and then shifted to $130^{\circ} \mathrm{C}$.

tive Stretch Relaxation Time. Phys Rev Lett 103:136001

Andreev M, Khaliullin RN, Steenbakkers RJA, Schieber JD (2013) Approximations of the discrete slip-link model and their effect on nonlinear rheology predictions. J Rheol 57(2):535-557

Bach A, Almdal K, Rasmussen HK, Hassager O (2003a) Elongational viscosity of narrow molar mass distribution polystyrene. Macromolecules 36: 5174-5179

Bach A, Rasmussen HK, Hassager O (2003b) Extensional viscosity for polymer melts measured in the filament stretching rheometer. J Rheol 47(2):429-441

Baumgaertel M, Schausberger A, Winter HH (1990) The relaxation of polymers with linear flexible chains of uniform length. Rheol Acta 29:400-408 


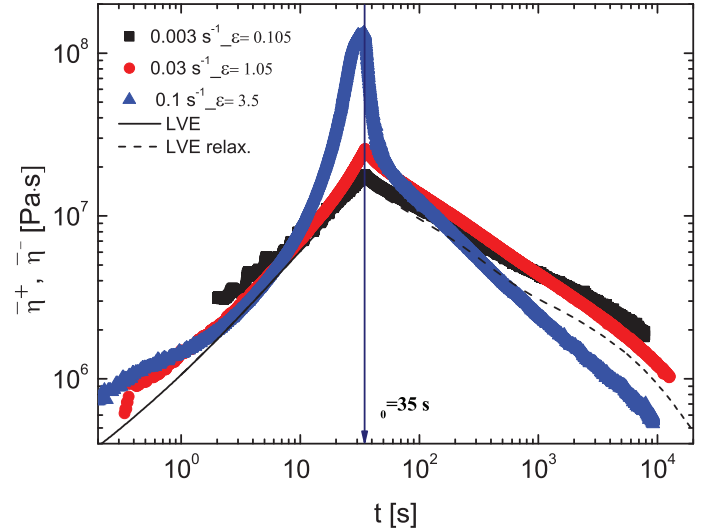

Fig. 5 Stress relaxation measurements of the Blend 50L-50S after a fixed flow time $t_{0}=35 \mathrm{~s}$ of uniaxial extension. The experiments were performed at $\dot{\epsilon}$ of $0.003,0.03$ and $0.1 \mathrm{~s}^{-1}$ at $130^{\circ} \mathrm{C}$. The solid line is the LVE prediction and the dashed line is the LVE relaxation prediction for $\dot{\epsilon}=0.003 \mathrm{~s}^{-1}$, both are based on the parameters listed in Table 5 .

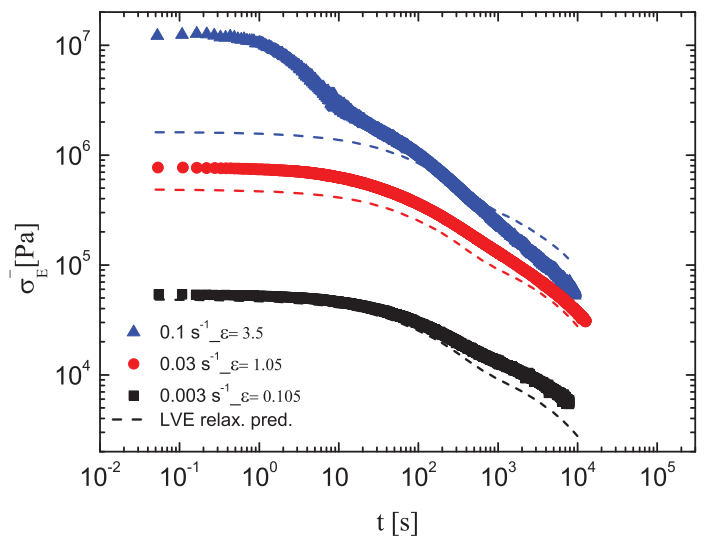

Fig. 6 The stress decay data after a fixed elongational flow time $t_{0}=35 \mathrm{~s}$ for the Blend 50L-50S. The data are originally from Figure 5 plotted according to Eq. 16.

des Cloizeaux J (1988) Double reptation vs. simple reptation in polymer melts. J Europhys Lett 5:437-432

de Gennes PG (1975) Dynamics of entangled polymer solutions. II Inclusion of hydrodinamic interactions. Macromolecules 9:594-598

Doi M and Edwards SF (1986) The Theory of Polymer Dynamics. Clarendon Press, Oxford

Hayes C, Bokobza L, Boué F, Mendes E, and Monnerie L (1996) Relaxation dynamics in bimodal polystyrene melts: a Fourier-transform infrared dichroism and small-angle neutron scattering study. Macromolecules 29:5036-5041

Huang Q, Alvarez NJ, Matzumiya Y, Rasmussen HK, Watanabe H, Hassager O (2013a) Extensional rheology of entangled polystyrene solutions suggests importance of nematic interaction. ACS Macro Letters 2:741-744

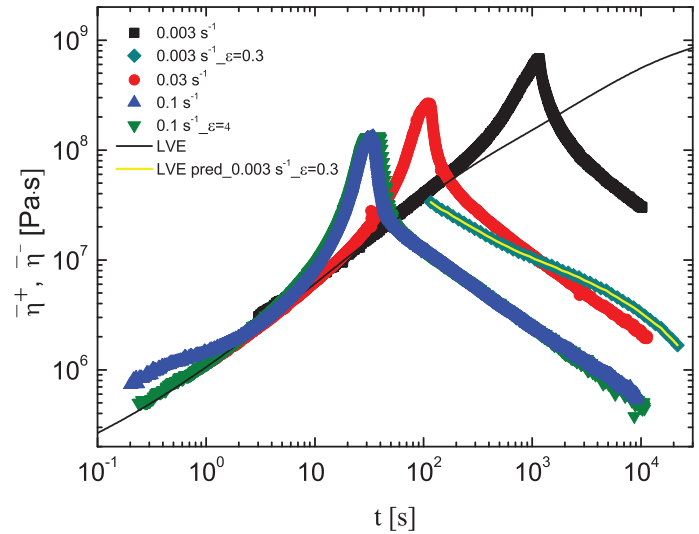

Fig. 7 Stress relaxation measurements of the Blend 50L-50S after fixed Hencky strain of $\epsilon_{0}=3.5$. The experiments were performed at $\dot{\epsilon}$ of $0.003,0.03$ and $0.1 \mathrm{~s}^{-1}$ at $130^{\circ} \mathrm{C}$. Two extra measurements have been done. One at $\dot{\epsilon}=0.1 \mathrm{~s}^{-1}$ where the flow has been stopped at $\epsilon=4$ and one at $\dot{\epsilon}=0.003 \mathrm{~s}^{-1}$ stopped at $\epsilon=0.3$, before entering the non linear regime.

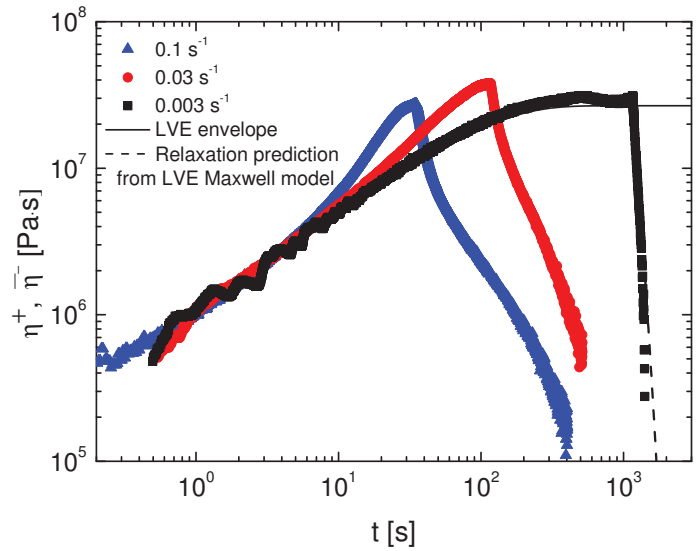

Fig. 8 Stress relaxation measurements of the pure PS-95k after fixed Hencky strain of $\epsilon_{0}=3.5$. The experiments were performed at $\dot{\epsilon}$ of $0.003,0.03$ and $0.1 \mathrm{~s}^{-1}$ at $130^{\circ} \mathrm{C}$. In all cases the flow was stopped at $\epsilon=3.5$ and allowed to relax until the force detected from the weight cell was almost zero.

Huang Q, Mednova O, Rasmussen HK, Alvarez NJ, Skov AL, Almdal K, Hassager O (2013b) Concentrated polymer solutions are different from melts: role of entanglement molecular weight. Macromolecules 46:5026-5035

Ianniruberto G, Brasiello A, Marrucci G (2012) Simulations of fast shear flows of PS oligomers confirm monomeric friction reduction in fast elongational flows of monodisperse PS melts as indicated by rheo-optical data. Macromolecules 45:8058-8066

Likhtman AE and McLeish TCB (2002) Quantitative Theory for Linear Dynamics of Linear Entangled Polymers. Macromolecules 35:6332-6343

Khaliullin RN, Schieber JD (2010) Application of the Slip-link Model to Bidisperse Systems. Macromolecules 


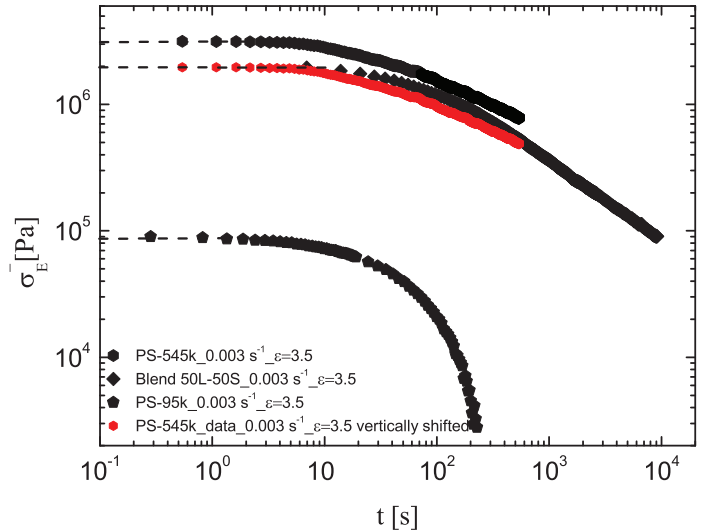

Fig. 9 Comparison of the stress decay data of the Blend 50L$50 \mathrm{~S}$, the pure PS-95k and the pure PS-545k at $130^{\circ} \mathrm{C}$ after cessation of elongational flow (up to $\epsilon=3.5$ ) at the same strain rate $\dot{\epsilon}=0.003 \mathrm{~s}^{-1}$. The dashed lines are guidelines meant to show the stress level right when relaxation starts. The red data are the one of the long chains PS-545k vertically shifted.

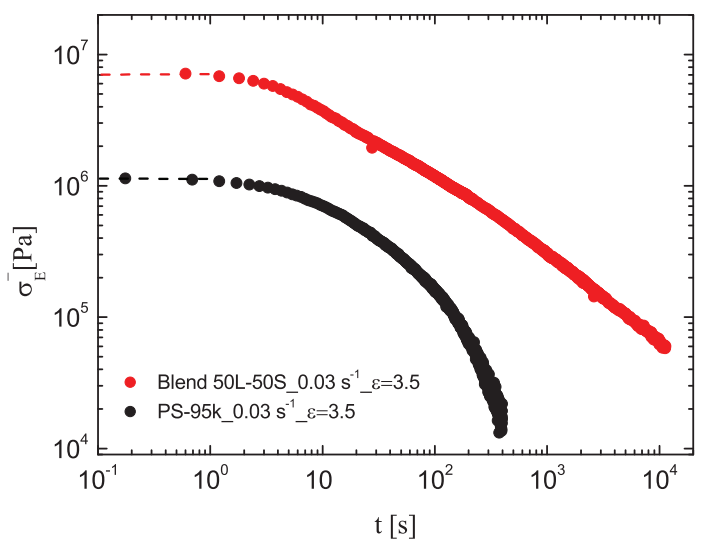

Fig. 10 Comparison of the stress decay data of the Blend 50L-50S and the pure PS-95k at $130^{\circ} \mathrm{C}$ after cessation of elongational flow (up to $\epsilon=3.5$ ) at the same strain rate $\dot{\epsilon}=0.03 \mathrm{~s}^{-1}$. The dashed lines are guidelines meant to show the stress level right when relaxation starts.

\section{3(14):6201-6212}

Kolte MI, Rasmussen HK, and Hassager O (1997) Transient filament stretching rheometer II: Numerical simulation. Rheol Acta 36(3):285-302

Kornfield, J. A., G. Fuller, and D. S. Pearson (1989) Infrared Dichroism Measurements of Molecular Relaxation in Binary Blend Melt Rheology. Macromolecules 22:13341345

Lee JH, Fetters LJ, Archer LA, Halasa AF (2005) Tube Dynamics in Binary Polymer Blends. Macromolecules 38:3917-3932

Lyhne A, Rasmussen HK, Hassager O (2009) Simulation of Elastic Rupture in Extension of Entangled Monodisperse Polymer Melts. Phys Rev Lett 102: 138301

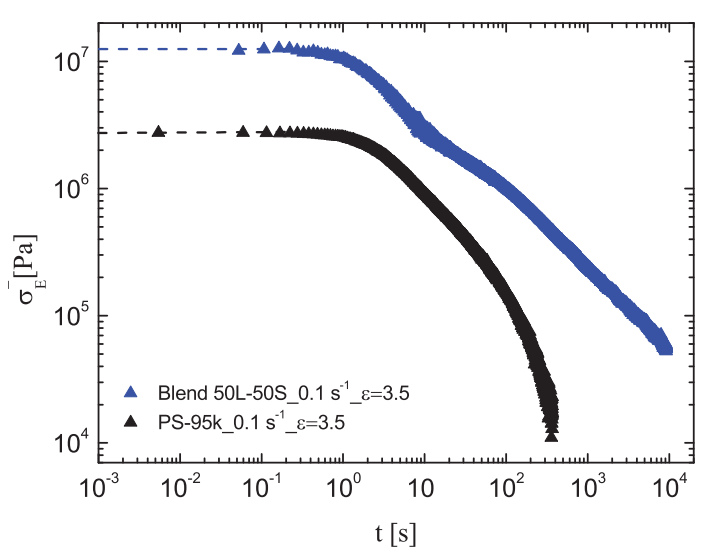

Fig. 11 Comparison of the stress decay data of the Blend 50L-50S and the pure PS-95k at $130{ }^{\circ} \mathrm{C}$ after cessation of elongational flow (up to $\epsilon=3.5$ ) at the same strain rate $\dot{\epsilon}=0.1 \mathrm{~s}^{-1}$. The dashed lines are guidelines meant to show the stress level right when relaxation starts.

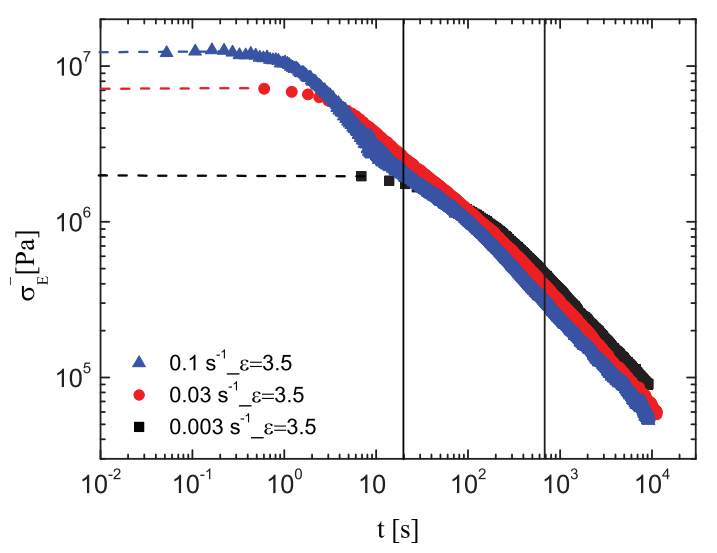

Fig. 12 The stress decay for the Blend 50L-50S. The data are originally from Figure 7 . The dashed lines are guidelines meant to show the stress level right when relaxation starts.

Marrucci G (1985) Relaxation by reptation and tube enlargement: A model for polydisperse polymers. J Polym Sci Polym Phys Ed 23:159-177

Milner ST, TCB McLeish (1998) Reptation and ContourLength Fluctuations in Melts of Linear Polymers. Phys Rev Lett 81:725-728

Ndoni S, Papadakis CM, Bates FS, Almdal K (1995) Laboratory-scale setup for anionic polymerization under inert atmosphere. Rev Sci Instrum 66: 1090-1095

Nielsen JK, Rasmussen HK, Hassager O, McKinley GH (2006)Elongational viscosity of monodisperse and bidisperse polystyrene melts. J Rheol 50:453-476

Nielsen JK, Rasmussen HK, Hassager O (2008) Stress relaxation of narrow molar mass distribution polystyrene following uniaxial extension. J Rheol 52(4):885-899

Park SJ, Larson RG (2004) Tube dilation and reptation in binary blends of monodisperse linear polymers. Macro- 
molecules 37:597-604

Rasmussen HK, Bejenariu AG, Hassager O, Auhl DJ (2010)Experimental evaluation of the pure configurational stress assumption in the flow dynamics of entangled polymer melts. J Rheol 54(6): 1325-1336

Rasmussen HK (2014)Interchain tube pressure effect in the flow dynamics of bi-disperse polymer melts. Rheol Acta 54:9-18

Read DJ, Jagannathan K, Sukumaran SK, Auhl D (2012) A full-chain constitutive model for bidisperse blends of linear polymers. J Rheol 56:823-873

Román Marín JM, Huusom JK, Alvarez NJ, Huang Q, Rasmussen HK, Bach A, Skov AL, Hassager O (2013) A control scheme for filament stretching rheometers with application to polymer melts. J Non-Newtonian Fluid Mech 194:14-22

Szabo P(1997) Transient filament stretching rheometer part I: Force balance analysis. Rheol Acta 36:277-284

Szabo P, McKinley GH (2003) Filament stretching rheometer: Inertia compensation revisited. Rheol Acta 42:269271

Struglinski MJ, WWGraessley (1985) Effect of polydispersity on the linear viscoelastic properties of entangled polymers. 1. Experimental observation for binary mixtures of linear polybutadiene, Macromolecules 18:2630-2643

Struglinski MJ, WW Graessley (1986) Effect of polydispersity on the linear viscoelastic properties of entangled polymers. 2. Comparison of viscosity and recoverable compliance with tube model predictions. Macromolecules 19:1754-1760

Tsenoglou C (1987) Viscoelasticity of binary polymer blends. ACS Polym Preprints 28:185-186

van Ruymbeke E, Masubuchi Y, Watanabe H (2012) Effective value of the dynamic dilution exponent in bidisperse linear polymers: from 1 to 4/3. Macromolecules 45:2085-2098

van Ruymbeke E, Nielsen J, Hassager O (2010) Linear and nonlinear viscoelastic properties of bidisperse linear polymers: Mixing law and tube pressure effect. J Rheol 54(5):1155-1172

van Ruymbeke E, Shchetnikava V, Matsumiya Y, Watanabe H (2014) Dynamic dilution effect in binary blends of linear polymers with well-separated molecular weights. Macromolecules 47:7653-7665

Wagner MH, Kheirandish S, Koyama K, Nishioka A, Minegishi A, Takahashi T (2005) Modelling strain hardening of polydisperse polystyrene melts by molecular by molecular stress function theory. Rheol Acta 44:235-243

Wagner M (2011)The effect of dynamic tube dilation on chain stretch in nonlinear polymer melt rheology. J of

Non-Newtonian Fluid Mech 166:915-924

Wang Y, Boukany P, Wang SQ, Wang X (2007) Elastic breakup in uniaxial extension of entangled polymer melts. Phys Rev Lett 99:237801
Watanabe H, Ishida S, Matzumiya Y, Inoue T (2004a) Viscoelastic and dielectric behavior of entangled blends of linear polyisoprenes having widely separated molecular weights: Test of tube dilation picture. Macromolecules 37:1937-1951

Watanabe H, Ishida S, Matzumiya Y, Inoue T (2004b) Test of full and partial tube dilation pictures in entangled blends of linear polyisoprenes. Macromolecules 37:6619-6631

Watanabe H, Matzumiya Y, Ruymbeke EV (2013) Component relaxation times in entangled binary blends of linear chains: reputation/CLF along partially or fully dilated tube. Macromolecules 46:9296-9312

Yaoita T, Takeharu I, Masubuchi Y, Watanabe H, Ianniruberto G, Marrucci G (2012) Primitive chain network simulation of elongational flows of entangled linear chains: stretch/orientation-induced reduction of monomeric friction. Macromolecules 45:2773-2782 